\title{
Nucleotides that are essential but not conserved; a sufficient L-tryptophan site in RNA
}

\author{
IRENE MAJERFELD, ${ }^{1}$ JANA CHOCHOLOUSOVA, ${ }^{2}$ VIKAS MALAIYA, ${ }^{1}$ JEREMY WIDMANN, ${ }^{3}$ \\ DANIEL MCDONALD, ${ }^{3}$ JENS REEDER, ${ }^{3}$ MATTHEW IYER, ${ }^{3}$ MALI ILLANGASEKARE, ${ }^{1}$ MICHAEL YARUS, ${ }^{1}$ \\ and ROB KNIGHT ${ }^{3,4}$ \\ ${ }^{1}$ Department of Molecular, Cellular and Developmental Biology, University of Colorado at Boulder, Boulder, Colorado 80309, USA \\ ${ }^{2}$ Institute of Organic Chemistry and Biochemistry, Academy of Sciences of the Czech Republic, Prague 6, Czech Republic \\ ${ }^{3}$ Department of Chemistry and Biochemistry, University of Colorado at Boulder, Boulder, Colorado 80309, USA \\ ${ }^{4}$ Howard Hughes Medical Institute, University of Colorado at Boulder, Boulder, Colorado 80309, USA
}

\begin{abstract}
Conservation is often used to define essential sequences within RNA sites. However, conservation finds only invariant sequence elements that are necessary for function, rather than finding a set of sequence elements sufficient for function. Biochemical studies in several systems-including the hammerhead ribozyme and the purine riboswitch—find additional elements, such as loop-loop interactions, required for function yet not phylogenetically conserved. Here we define a critical test of sufficiency: We embed a minimal, apparently sufficient motif for binding the amino acid tryptophan in a random-sequence background and ask whether we obtain functional molecules. After a negative result, we use a combination of three-dimensional structural modeling, selection, designed mutations, high-throughput sequencing, and bioinformatics to explore functional insufficiency. This reveals an essential unpaired $G$ in a diverse structural context, varied sequence, and flexible distance from the invariant internal loop binding site identified previously. Addition of the new element yields a sufficient binding site by the insertion criterion, binding tryptophan in 22 out of 23 tries. Random insertion testing for site sufficiency seems likely to be broadly revealing.
\end{abstract}

Keywords: SELEX; aptamer; evolution; tryptophan

\section{INTRODUCTION}

Defining minimal sequence requirements for specific RNA activities is the key to many basic problems in RNA structure and function. Understanding how nucleotides produce specific catalytic or binding effects (Ellington and Szostak 1990; Bartel and Szostak 1993), predicting the secondary or tertiary structure of the active region (Harris et al. 1994; Anderson and Mecozzi 2005a,b), testing whether a motif contains an over-representation of sequences relevant to the genetic code (Yarus and Welch 2000; Yarus et al. 2009), calculating the probability of finding the motif either in random-sequence pools in SELEX experiments or in wholegenome scans (Sabeti et al. 1997; Bourdeau et al. 1999; Knight and Yarus 2003; Knight et al. 2005; Laserson et al. 2005; Legiewicz et al. 2005; Kim et al. 2007; Kennedy et al.

Reprint requests to: Rob Knight, Department of Chemistry and Biochemistry, University of Colorado at Boulder, UCB 215, Boulder, CO 80309, USA; e-mail: rob@spot.colorado.edu; fax: (303) 492-7744.

Article published online ahead of print. Article and publication date are at http://www.rnajournal.org/cgi/doi/10.1261/rna.2220210.
2010), as well as the relation between site complexity and function (Carothers et al. 2004, 2006), all depend on an accurate census of an active RNA site. Traditionally, this characterization has been performed by comparison of related sequences and mutagenesis of one or a few functional sequences. The assumption underlying these techniques is that unimportant residues are free to vary, whereas residues critical for function will be preserved.

An illustration of necessary but not sufficient sequence elements occurs for the hammerhead ribozyme under physiological conditions. Minimal hammerhead ribozymes, thoroughly characterized by NMR and X-ray crystallography, are active under conditions of high magnesium but inactive with physiological divalent concentrations. A series of elegant experiments by Khvorova and colleagues demonstrated that a distal loop-loop interaction is required for activity under physiological conditions (Khvorova et al. 2003; Penedo et al. 2004; Saksmerprome et al. 2004). However, because many different sequences can satisfy the requirements for the distal interaction, the relevant nucleotides were not included in the original active site specification (McCall et al. 1992). 
In this study, we explore a new method for testing whether an RNA site is sufficient. We embed the site in a random-sequence background and retest for function. If all sequence and structural elements required for function were present in the site specification, we would predict a large fraction of the resulting sequences to show full activity (100\% function is not necessarily expected because of the effects of sequences outside the active site; e.g., on folding) (Knight et al. 2005). If the site specification is incomplete, we instead expect that most sequences would be inactivea small fraction of active sequences may spotlight additions necessary for a sufficient active structure.

Here we focus on the minimal L-Trp-binding motif. This site has been independently selected 56 times in four different random-sequence backgrounds and is highly likely to be the minimal RNA structure that specifically binds the amino acid tryptophan (Majerfeld and Yarus 2005; Yarus et al. 2009). We show that a combination of SELEX, molecular modeling, and testing of specific mutant constructs define an unexpected, nonconserved piece of the motif, and that incorporation of these additional constraints enabled generation of functional Trp-binding sequences in randomized contexts (Fig. 1), that is, a sufficient site. We chose the Trp motif for small size, stability, substantial affinity for its ligand $\left(K_{\mathrm{d}} \approx 12 \mu \mathrm{M}\right)$, and relevance to the evolution of the genetic code (Majerfeld and Yarus 2005; Yarus et al. 2009). However, we expect these techniques to be applicable to any comparable RNA motif.

\section{RESULTS}

\section{Failure of initial insertions into randomized contexts}

Initial computer-generated sequences, in which the previously defined Trp binding-site motif was inserted into a randomized but supposedly congenial RNA fold, all exhibited weak or negligible tryptophan affinity. Binding profiles of these composite sequences (Fig. 2B,C) contrast remarkably with the binding and Trp affinity elution of selected aptamers (Fig. 2A). Figure 2 in fact contains only a small part of the data. None of seven composite sites like Trp 4 and 602 showed activity like that in Figure 2A; instead, the chromatographic peak near the void contained almost $100 \%$ of the applied transcript.

Further, the difficulty is unlikely to be simply related to misfolding. Comparison of Trp 4 (Fig. 2B) and Trp 602 (Fig. 2C) shows that more stable flanking helices, which in principle force the bracketed internal loop of the active binding site more unequivocally, reduce the minuscule binding observed. Clearly, the initial Trp site consensus fails the random insertion test of sufficiency.

Our attempt to improve activity by forcing the formation of the conserved internal loop had eliminated nearby features in the selected molecule in favor of lengthened flanking helices. It was a reasonable hypothesis, therefore,

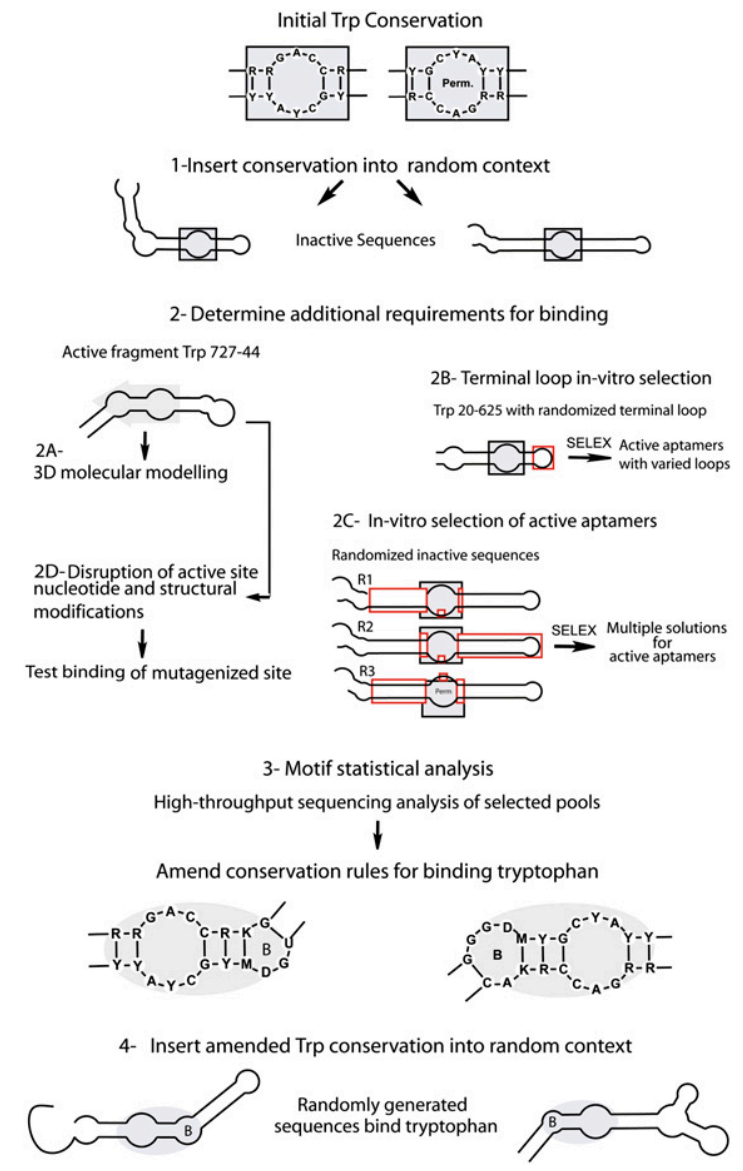

FIGURE 1. Overview of experimental procedures. See the text for details.

that these constructions removed an essential nearby structure we had not appreciated, and thereby depressed site function. We set out to detect this (then) hypothetical structure (outlined in Fig. 1).

\section{Three-dimensional molecular modeling}

After the failure of the initial insertion experiments, we explored Trp site structure and function using computational methods. Three-dimensional (3D) models of a functional, typical Trp site ( $\left.\operatorname{Trp} 727-44 ; K_{\mathrm{d}} \approx 15 \mu \mathrm{M}\right)$ (Fig. 6A, below) and a nonfunctional derivative (Trp 727-28; a 28-nucleotide [nt]-long RNA with the canonical Trp main loop flanked by featureless helices and a terminal tetraloop) were prepared, based on a multiple sequence alignment, multiple singlesequence folds, and probing data. A three-dimensional (3D) model of the aptamer (Trp 727-44) with tryptophan docked in the expected binding site was also studied.

\section{Molecular dynamics and normal mode analysis}

Molecular dynamics trajectories of $1 \mathrm{nsec}$ were carried out for both Trp 727-44 and Trp 727-28. These trajectories 

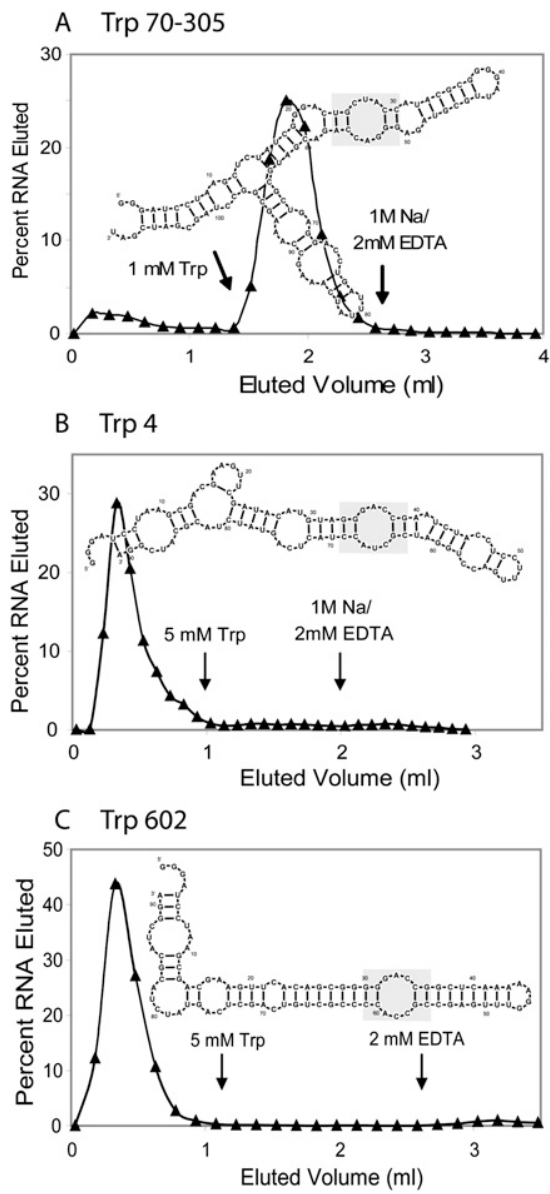

FIGURE 2. Secondary structure and affinity chromatography of a tryptophan aptamer and randomly generated sequences. $(A)$ Aptamer isolated from a $70 \mathrm{~N}$ nucleotide-long randomized region in an in vitro selection (Majerfeld and Yarus 2005). (B) Randomly generated sequence containing the initially recognized conservation. (C) As in $B$, but sequence has longer stems for increased internal loop stability.

were inspected visually for differences in RNA motions. Normal mode analysis of the two systems was also performed, and the motions were compared visually. The main differences appeared in the flexibility originating in the asymmetric 3-1 internal GGG:A loop of active Trp 72744. The main internal loop in Trp 727-44 could open up, and interact with, the secondary internal loop. In contrast, the inactive $\operatorname{Trp} 727-28$ structure appeared to remain closed at the binding site, potentially hindering tryptophan access. Molecular dynamics up to $4 \mathrm{nsec}$ with tryptophan in the site were consistent, suggesting that the main and secondary GGG:A loops can approach and perhaps interact. Moreover, when such a loop-loop interaction is forced by harmonic constraints between the GG nucleotides from the GGG:A internal loop and AC nucleotides from the main binding site, the resulting structure agreed with protection and interference experiments: A from the CCA of the binding site is unpaired and more exposed to external interactions (enhancement) (Majerfeld and Yarus, 2005), while A and $C$ nucleotides from the GAC loop are blocked by hydrogen bonding (protection) (Majerfeld and Yarus, 2005). An interference occurs on CC (CCA) and AC (GAC). Thus, a hypothetical interaction between the new G-containing loop and the previous site was potentially consistent with known site properties.

\section{Consistency with variety in the initial pools}

To evaluate the specific hypothesis that the somewhat variable second loop was a crucial element, we reanalyzed previously collected data after SELEX (Majerfeld and Yarus 2005). Aligning and folding the selected sequences to the randomly generated inactive sequences and directing the search to the region close to the main binding site loop, we found an unpaired $G$ upstream of the CYA module in the functional sequences, but not in our inactive sequences. As shown explicitly for a sample of sequences in Figure 3, all selected sequences indeed had a looped G 3-6 nt $5^{\prime}$ of the CYA of the major loop. Because the $G$ varied both in position and structural context, it was not initially identified as part of the binding site (Majerfeld and Yarus 2005).

\section{Secondary loop structure is not conserved}

Trp 20-625 (Fig. 4) is an aptamer isolated in an in vitro selection from a 20-nt-long randomized tract (Majerfeld and Yarus 2005). The limited sequence space available (the first and last of the 20 originally random nucleotides are indicated in Fig. 4A) suggests that additional components complementing the main loop function would be forced into the hairpin loop, even though other selection isolates, related to Trp 20-625, had varied pentaloops. In fact, randomization of only the five loop nucleotides alone resulted in loss of binding. Starting from a pool based on Trp 20-625 (shortened at 5' and 3' ends) with a randomized pentaloop (see Materials and Methods), two rounds of selection resulted in a peak of $13 \%$ of RNA eluted by L-Trp. The eluted peak was cloned and sequenced. Figure $4 \mathrm{~B}$ summarizes the results: $90 \%$ of the resulting sequences were pentaloops with UNNGG, UHRCG, and GHMAG. All selected pentaloops contain a $\mathrm{G}$ that is $4 \mathrm{nt} 5^{\prime}$ to the $\mathrm{C}$ of the conserved CYA of the main loop. A second G, $5 \mathrm{nt}$ away, is present in $48 \%$ of sequences. This selection strongly supports a requirement for unpaired G upstream of CYA at a defined range of distances. However, the vicinity of the secondary $G$ is unexpectedly structurally flexible. The $G$ context can be a hairpin, bulge, or internal loop (see below).

\section{Selections that create a secondary loop}

An inactive, randomly generated sequence that contained the 14 conserved nucleotides of the main CYA loop (Fig. 2C) was modified by suitable PCR primers (see Materials 


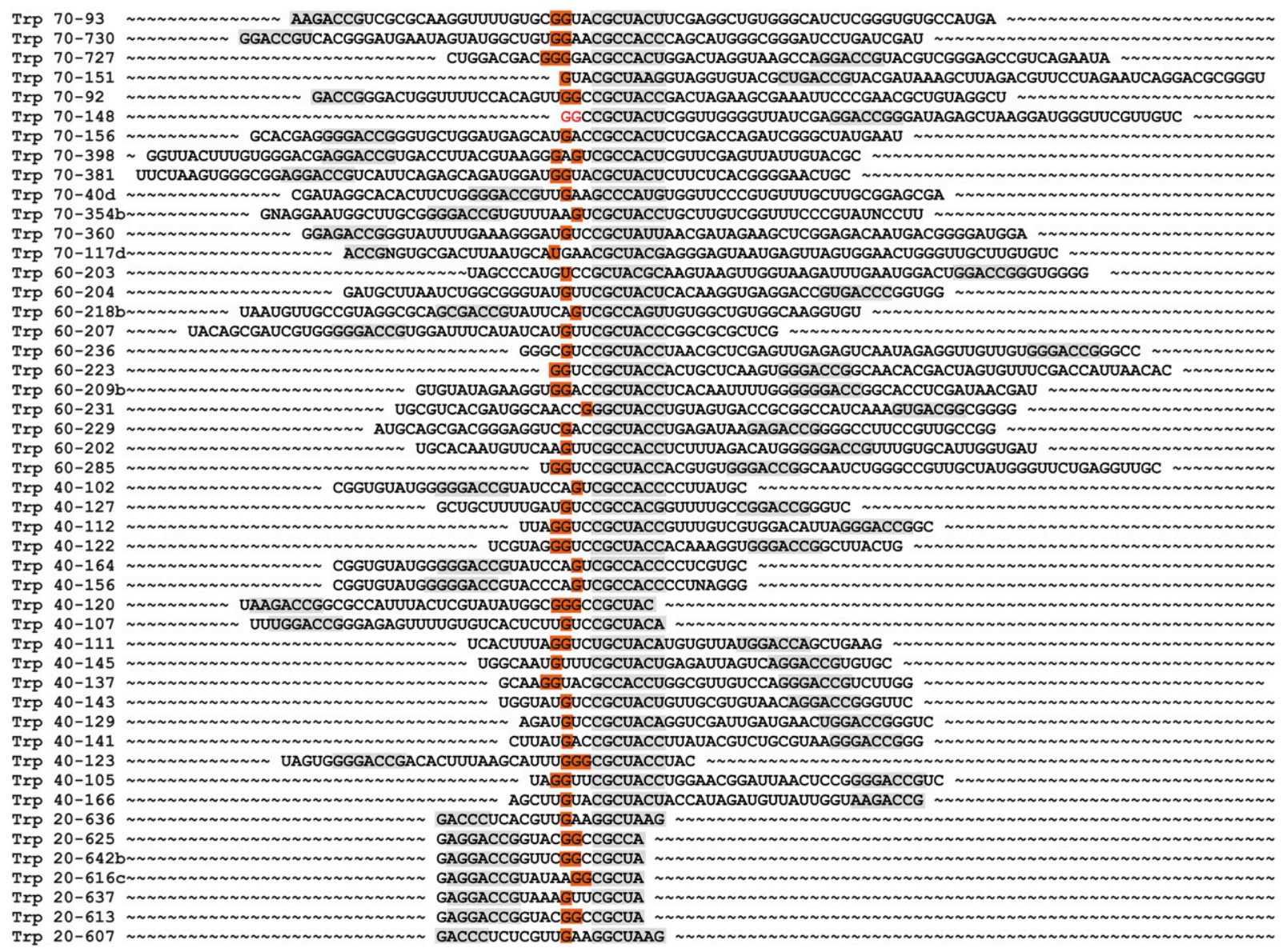

FIGURE 3. Forty-eight initial Trp-binding sequences selected from $70,60,40$, and $20 \mathrm{nt}$ randomized tracts. Sequences are aligned on the conserved major CYA loop sequence. All conserved nucleotides are shown on a gray background, with potential essential G's on a red background when in initially randomized positions, or as a red letter when it was derived from fixed nucleotides. These sequences are taken from the four published selections (Majerfeld and Yarus 2005).

and Methods). This construct, as well as a second construct that differed only in the permutation of the main tryptophan binding site, did not have any detectable binding activity. They were randomized within the main binding site according to its conservation (Majerfeld and Yarus 2005) and also at either side of the main binding loop, as shown in Figure 5A (R1, R2, and R3). Randomized RNA transcripts were selected to isolate from randomized regions additional structures needed for tryptophan binding. In cycles 2 (selection R2) or 3, each of the three independent selections succeeded with elution peaks of $30 \%-45 \%$ of applied RNA; those pools were cloned and sequenced. Examples of sequences obtained are presented in Figure $6 \mathrm{~B}$ - in varied, sometimes unanticipated ways, all selected RNAs acquire a secondary loop that presents a $G 5^{\prime}$ proximal to CYA.

In selection R1, a loop could not be generated $5^{\prime}$ to CYA in a straightforward fashion because that side of the main loop was not randomized. Nevertheless, of the 17 sequences obtained from this selection, 10 molecules added a looped G nucleotide 4 or $5 \mathrm{nt} 5^{\prime}$ to CYA by mutational addition or deletion. One of these sequence insertions, LB 322, is shown in Figure 5B. Five sequences, instead, deleted C36 (LB 331 in Fig. 5B) to bulge the paired G. The remaining two sequences deleted $5 \mathrm{nt}$, U47 to G51, a change that can

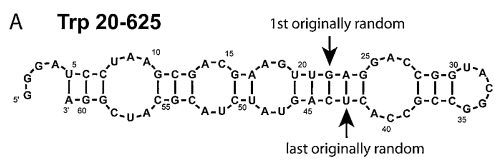

Selected Pentaloops

\begin{tabular}{ccc}
\hline $\begin{array}{l}\text { Loop--Stem } \\
\text { GAC CGG }\end{array}$ & Pentaloop & Stem--Loop \\
CCG & CCA & No.sequences \\
\hline U NN GG & $33(46 \%)$ \\
U HR CG & $17(24 \%)$ \\
G HM AG & $14(20 \%)$ \\
V WW NG & $7(10 \%)$ \\
\hline Total & & 71
\end{tabular}

FIGURE 4. Selection of active sequences by randomization of a hairpin loop. (A) Selection scheme showing the initially randomized region. (B) Selected pentaloops from loop re-randomization, showing the fraction of sequences with each pattern at the five tested positions. 
A

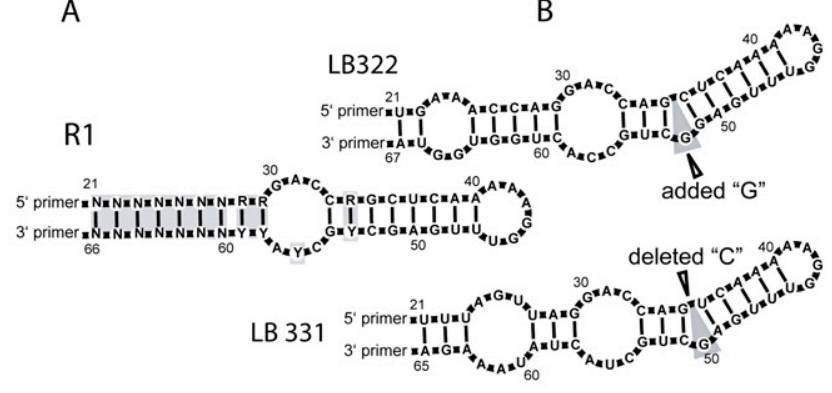

R2

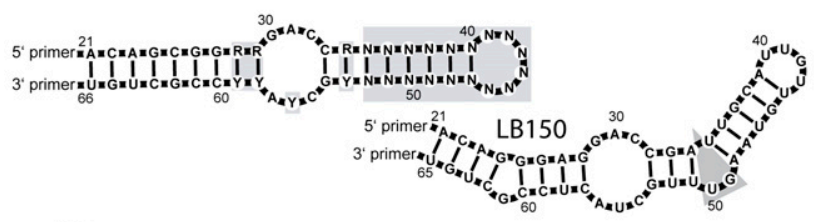

R3

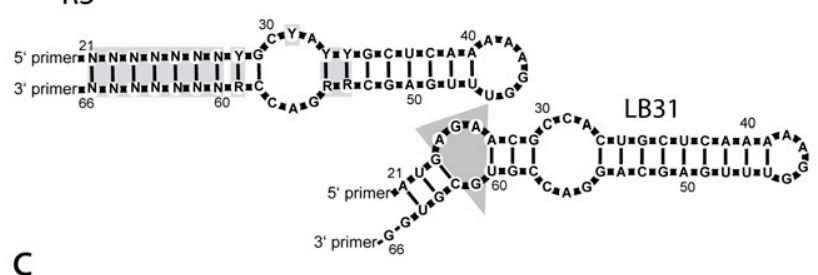

Selection R2

LB52 ACAGCGGAAGACCGUGGUUAGUGAAGAUCGUACGCUAUUCCGCUGU LB109a ACAGCGGAAGACCGGGAGCCAGGCAGGUUUGACGCCACUCCGCUGU LB135 ACAGCGGAAGACCGUGUAGAUUAAUCUAAGGACGCUACUCCGCUGU LB141 ACAGCGGAAGACCGAGAUCUAAGUGAUAUGUUCGCCACUCCGCUGU LB104 ACAGCGGAAGACCGGGAUCUGGAUAGAUUGGCCGCUAUCCCGCUGU LB132 ACAGCGGAAGACCGUGACCUGAAGGGGUCGAACGCUAUUCCGCUGU LB133 ACAGCGGAAGACCGACAAUGGGAACAGAAGGGCGCUAUUCCGCUGU LB136 ACAGCGGAAGACCGAGGGCCGGUAGGUCAGAUUGCUACUCCGCUGC LB154 ACAGCGGAGGACCGUUGGAUAUUUACUCAGUUCGCUACUCCGCUGU LB128 ACAGCGGAGGACCGUAAGCAAAUAAUCGAAGUCGCCACUCCGCUGU Selection R3

LB2 GAAUGGACGCCACCGCUCAAAAAGGUUUGAGCGGGACCGUGUUCGU LB45 CCUGGUACGCUACCGCUCAAAAAGGUUUGAGCAGGACCGUCAG LB31 AUGAGAACGCCACUGCUCAAAAAGGUUUGAGCAGGACCGUGCGUGG LB59 GUGAGGGCGCCACUGCUCAAAAAGGUUUGAGCAGGACCGUGGGUAC LB4b GUCGAGUCGCUACUGCUCAAAAAGGUUUGAGCAGGACCGUUGUACG LB32 GACAGUUCGCCACUGCUCAAAAAGGUUUGAGCAAGACCGAUGUUGG LB33 CCGUAGUCGCCACCGCUCAAAAAGGUUUGAGCGGGACCGUAUCGGC LB43 UAGGAGCCGCCAUUGCUCAAAAAGGUUUGAGCAAGACCGUAUUAGU LB69b GGUAGUACGCUACCGCUCAAAAAGGUUUGAGCGAGACCGUUGCCGA

FIGURE 5. Secondary loop selection. (A) Initially randomized structures for selections R1, R2, and R3. (B) Example foldings from the corresponding final pools. Partial alignment of sequences from selections R2 and R3 with "G" highlighted.

produce a symmetrical CU:GU internal loop. These active PCR mutations strongly suggest that a single bulged G 4-5 nt $5^{\prime}$ of $\underline{C} C A$ is the simplest route to an active structure.

Selections R2 (50 sequences) and R3 (62 sequences) were mutagenized in the secondary loop region, and yielded a variety of structures with unpaired $\mathrm{G} 5^{\prime}$ to the CYA module. As was the case for aptamers isolated from fully randomized pools, GU nucleotides were predominant in these loops, followed by GG and GA. Two predicted foldings are shown (Fig. 5B), and a sample of active sequences with their unpaired G's highlighted is displayed in Figure 5C.

\section{Disruption of active sites}

Mutational disruption of active binding sites should confirm the identity of the essential looped $\mathrm{G}$ identified above. Trp 727-44 Fig. 6A is an active fragment of an initial selected aptamer, Trp 70-727. In addition to the main tryptophan loop site, it contains a second internal loop. We introduced changes in the second loop, and tested their effect by affinity chromatography. Figure $6 \mathrm{~B}$ shows the affinity behavior of $\operatorname{Trp}$ 727-44 and mutationally related constructs, active RNAs on the left and inactive on the right. The Bayesfold (Knight et al. 2004b) predicted structure of Trp 727-44 and mutationally related constructs are shown in Figure 6A, with active RNAs on the left and inactive on the right. For active constructs, $60 \%-80 \%$ of applied RNA binds and elutes immediately upon addition of $1 \mathrm{mM}$ tryptophan. No further RNA is eluted by increased amino acid or by a high salt-EDTA wash. The second loop is required for binding because its removal (RNA M-1) completely abolishes affinity. Equally damaging was the permutation of the conserved nucleotides in the main binding loop, CGCCACU and AGGACCG, (RNA M-6), so the orientation of the main loop relative to surrounding sequences is essential. However, the secondary loop could be changed to a GG (RNA M-2) or GA (RNA M-3) bulge, though not to UA (RNA M-7). The G-containing bulge could be reduced to a single $\mathrm{G}$ (structure M-5) but not to a single, unpaired A in the same molecular context (RNA M-8). These mutational results decisively confirm the requirement for, at minimum, an unpaired $\mathrm{G} 5^{\prime}$ to the main CCA — the resulting structures, though varied, are similarly functional (Fig. 6B).

\section{Revised definition of the Trp binding site, and new sequences incorporating second loop structure}

Using all information gathered about the second loop from these mutational and SELEX experiments, we performed pyrosequencing on the original Trp selections to gather an additional 37,133 sequences (see Materials and Methods) and set out to redefine the motif using bioinformatics. We filtered all sequences to remove noise from PCR, the sequencing procedure itself, and the intentional mutation in the $70 \mathrm{~N}$ selection (Quince et al. 2009). We strictly looked for foldings that contained the GAC/CYA with the secondary bulge adjacent. From this restricted set of foldings, we generated a refined consensus information of the Trp site.

Based on iterative refinement of the pattern with example sequences and predicted secondary structures in the set that either did or did not match the pattern, we redefined the tryptophan binding site as follows (Fig. 7A). The site consists of a main internal loop and an accessory bulge, internal loop, or terminal loop. The second loop is separated from the main loop by two to four conserved base pairs. The previously described main loop, GAC/CYA, is framed by conserved nucleotides on both sides (Majerfeld and Yarus 2005). The conserved nucleotides $5^{\prime}$ to CYA 
A

Trp 727-44

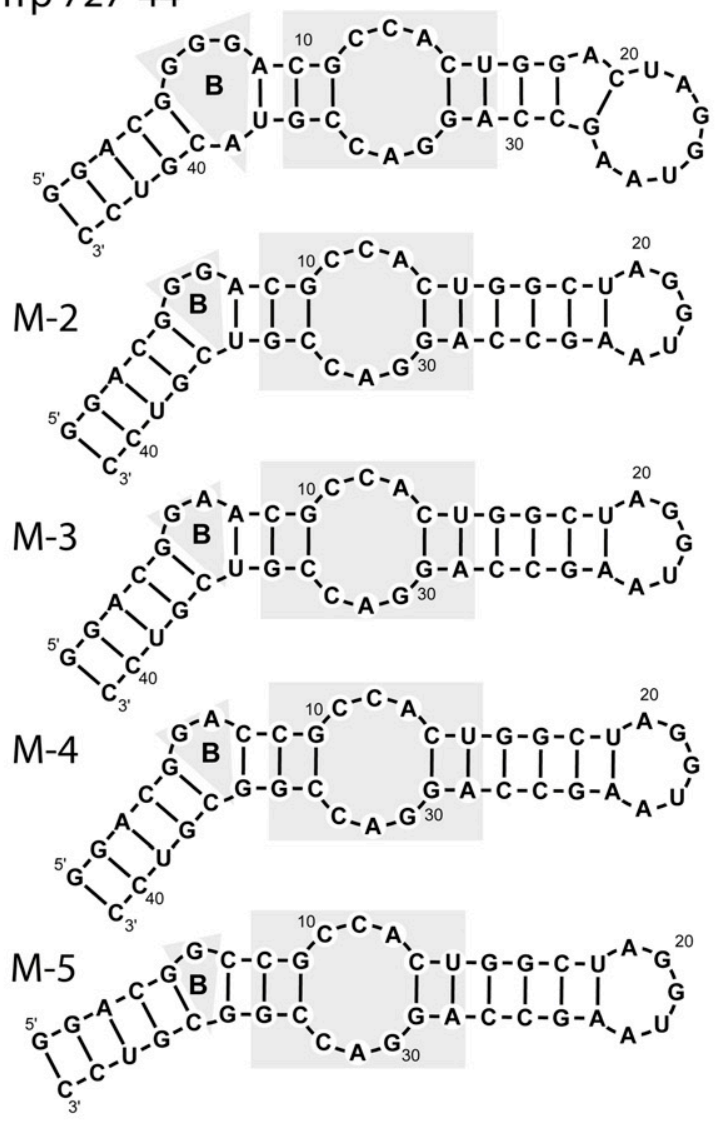

B

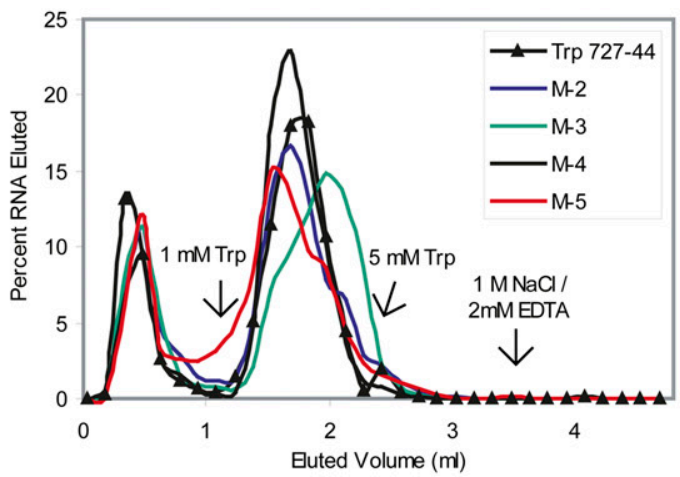

$M-1$
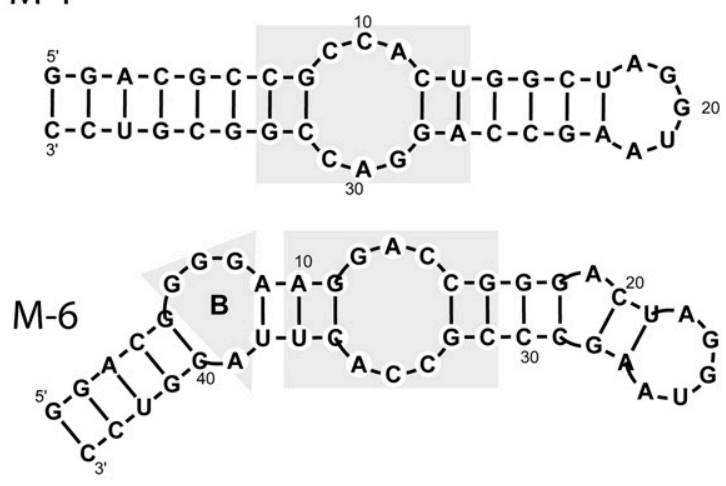

M-7
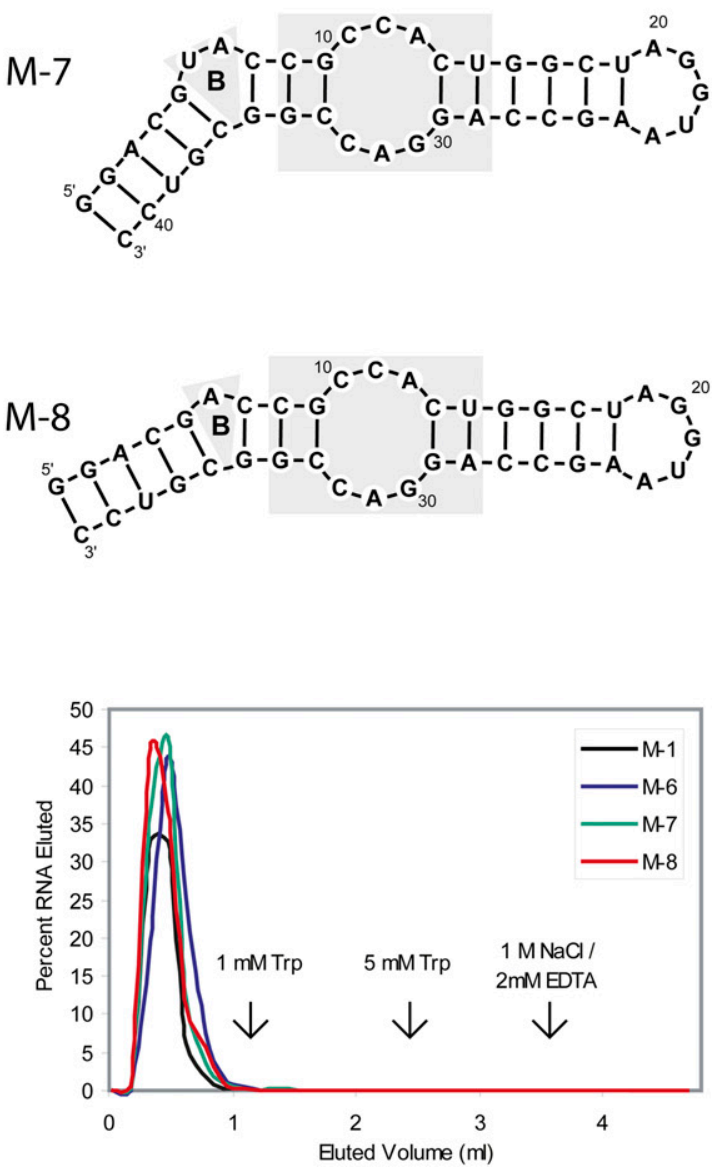

FIGURE 6. Mutagenic analysis of an active sequence, Trp 727-44. $(A, B)$ Active sequences are on the left, with affinity profiles below, and inactive mutants are on the right, with their affinity tests below.

form part of the intermediate stem for Loop B, which varies in size and nucleotide composition with a minimum requirement of one unpaired "G" 3-6 nt 5 ' to the "C" of CYA. Although one unpaired " $G$ " is sufficient for binding tryptophan, it may not be the optimal solution; in all selections from fully randomized segments $2 / 0$ bulges (GU, $\mathrm{GA}$, and GG) were most frequent. When three or more unpaired nucleotides appear, they form an internal loop rather than a bulge.

\section{Sequences that are in fact sufficient}

Given a new site definition that embodies a more complex idea of the tryptophan binding site consistent with the 
A New conservation of the Trp site
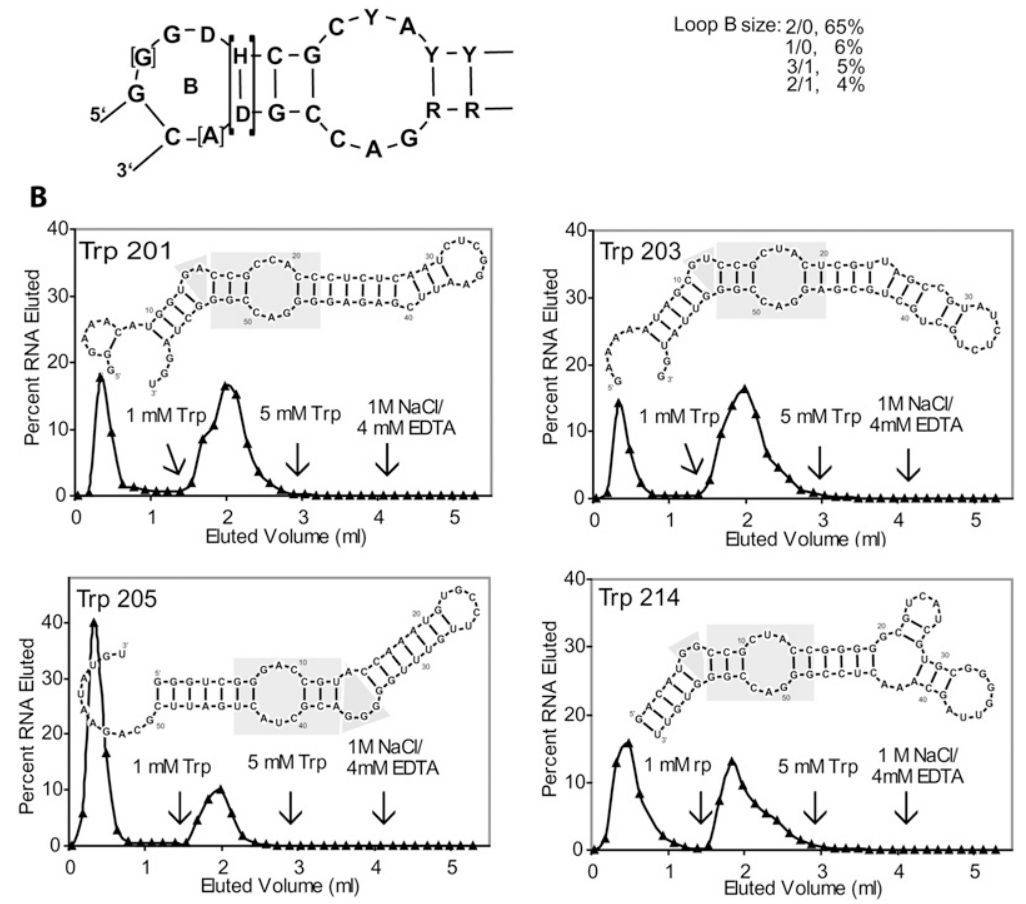

FIGURE 7. (A) A newly defined tryptophan binding site derived from selection, construction, mutagenesis, and massed sequence analysis of selected pools. The binding site illustrates conservation with a cutoff of $80 \%$. (B) Secondary structure and affinity chromatography of four out of 23 tested randomly generated sequences containing variations of the illustrated binding site.

above observations (Fig. 7A), we again performed the test of inserting sites into randomized contexts. Tests included both CCA and CUA sequences in the main loop, and in both $5^{\prime}$ '3'permutations, with secondary bulges GU, GA, GG, as well as internal GGG/A loops. Figure 7B shows four successful elution profiles; 22 of 23 of these trial insertions were stably bound to a Trp-Sephadex column and then eluted with $1 \mathrm{mM}$ L-Tryptophan. We conclude that Figure 7A is finally a sufficient tryptophan site. Later, we will summarize the effect of these changes on the argument for a stereochemical origin of the genetic code, though the substantially conserved main loop Trp anticodon (Majerfeld and Yarus 2005; Yarus et al. 2009) is unaltered here.

\section{DISCUSSION}

Initial sequence analysis did not detect all essential parts of the tryptophan binding motif because the second loop defined here is essential, but variable. The initial evidently conserved region, the CYA-GAC internal loop, was correctly confirmed by protection and interference experiments as indispensable for binding activity. Thus, the central site was well supported by the usual kinds of evidence, although, as we show by the random insertion test, it is insufficient for Trp binding.
When we tested the sufficiency, as well as the necessity for Trp affinity by inserting artificial Trp aptamer sequences into random tracts, the single loop model failed. To our surprise, even more specifically forced loops showed no binding at all. We suspected (Fig. 1) that the answer lay in a missing tertiary element and studied the structure by molecular modeling, comparing the structure and dynamics of models of active and inactive sites. From this point, we suspected that a small internal loop interacts with the main binding site. We therefore performed more detailed statistical analysis of randomly generated, inactive sequences and active sequences isolated from SELEX. Specifically, we looked anew at selected RNAs and their calculated secondary structures, concentrating on a secondary loop in the vicinity of the main loop, $5^{\prime}$ from the main internal loop CCA. A required, indeed, a universal feature had escaped notice. Mutagenesis of this new feature in active molecules and selection of activity in regionally randomized molecules invariably confirmed an essential looped $G$ as small as a single bulged nucleotide.

Thus, our major finding is that essential ribonucleotides can have unexpected properties:

- They can vary in number, forming loops of 1-5 nt.

- They can vary in sequence, several sequences being similarly active, including $\mathrm{G}$ alone.

- They can occur in differing structures; here hairpin, internal, and bulge loops.

- They can vary in distance from the main site; here 3-6 nt 5' of CYA.

Because such variations occur simultaneously in an unknown site, such functional nucleotides become elusive to normal criteria of sequence or structural conservation. None of this seems improbable once an example is in hand, yet the net implication - invisible essential nucleotides-seems a notable addition to the concept of a "sufficient" RNA active site.

Finally, the second loop in the Trp site has several specific implications. First, previous estimates of the probability of finding particular types of RNA sites (Knight and Yarus 2003; Knight et al. 2005; Kennedy et al. 2008) may be inflated by failing to take into account undetectable, but nonetheless important parts of the active site, such as those revealed here. Second, embedding the site in a random-sequence background provides an effective means for detecting such 
nonconserved active-site participants. Finally, multipart sites such as the Trp site, including essential pieces linked by partially elastic rules may be common. Their flexibility elevates their frequency in random-sequence pools with respect to uniquely defined sites of similar size. This combinatorial argument applies equally to the evolution of biologically active sequences and also to RNAs freshly selected in vitro. Accordingly, we suggest that insertion testing might reveal hitherto unsuspected elements, even in wellcharacterized natural and selected RNA active sites, including ribozymes.

\section{MATERIALS AND METHODS}

\section{Overview}

An overview of the experimental approach is shown in Figure 1. We initially generated sequences as previously described using the published minimal Trp active site (Majerfeld and Yarus 2005). Briefly, for the sequence NYGCYAYYNNRRGACCRN, with 3 base pairs connecting the unspecified residues upstream of or downstream from the site, we generated a sequence that could fulfill the requirements using equal frequencies of the four bases (Fig. 1, Step 1). We then used the RNAfold program in the Vienna package (Hofacker et al. 1994) to verify that that the relevant base pairs formed, and that the CYA and GAC that comprise the minimal internal loop remained unpaired (Fig. 1, Step 2B). When these sequences appeared inactive, we hypothesized that the issue was inefficient folding, and we regenerated the sequences using very long flanking helices (Fig. 1, Step 2C). When these sequences were also inactive, we re-analyzed Trp sequences from SELEX, making a series of constructs based on the original sequence that added or deleted specific elements implicated in function either through molecular modeling or comparative sequence analysis (Fig. 1, Step 2A). Additionally, we performed selections starting with the inactive sequences with long flanking helices, mutagenizing either the upstream part or the downstream helix relative to the CYA motif (Fig. 1, Steps 2,3) to reveal parts of the molecule relevant for activity. Finally, combining data from these experiments and high-throughput sequencing, we generated a new site specification whose insertion produced functional sequences (Fig. 1, Step 4).

\section{Preparation of affinity matrix}

Fmoc-protected tryptophan was coupled to EAH Sepharose (Amersham) via its carbonyl group as previously (Illangasekare and Yarus 2002), except that pentafluorophenyl ester (OPfp) preactivated Fmoc aminoacid was used. The result was two matrices at $1 \mathrm{mM}$ and $2 \mathrm{mM}$ tryptophan. Acetylated Sepharose was prepared by reaction of the same Sepharose with a 30-fold excess of acetic anhydride (with respect to resin amino groupings).

\section{Affinity chromatography and selection procedures}

For the terminal loop selection, Trp 20-625 (Fig. 4) was shortened at both ends and minor nucleotide changes were introduced. Affinity columns for binding tests were $0.3 \mathrm{~mL}$; those used for in vitro selections were $0.4 \mathrm{~mL}$. Column chromatography buffer was $50 \mathrm{mM}$ Hepes ( $\mathrm{pH} 7.0$ ), $250 \mathrm{mM} \mathrm{NaCl}, 5 \mathrm{mM}$ each $\mathrm{MgCl}_{2}$ and $\mathrm{CaCl}_{2}$. Elution buffer also contained $1 \mathrm{mM}$ tryptophan. Selections were performed as previously described (Majerfeld and Yarus 2005). In selections R1, R2, and R3, cycles 2 and 3 were preceded by counterselection through acetylated Sepharose.

The initial DNA sequences (T7 promoter underlined) were as follows:

- Terminal loop selection: taatacgactcactataggccgcagaagttgaggac cgg(N5)ccgccactcagtatcatcggat;

- Selection R1: taatacgactcactataggcatgtagacgcaactcac(N7)RRgacc RgctcaaaaaggtttgagcYgcYaYY(N7)ggagtagtgcaggagatgtg;

- Selection R2: taatacgactcgactataggcatgtagacgcaactcacacagcggRR gaccR(N18)YgcYaYYccgctgtggagtagtgcaggagatgt; and

- Selection R3: taatacgactcactataggggcatgtagacgcaactcac(N7)Ygc YaYYgctcaaaaaggtttgagcRRgaccR(N7)ggagtagtgcaggagatgtg.

PCR primers for the terminal loop selection were nucleotides 1-19 and 31-49. PCR primers for selections R1, R2, and R3 were nucleotides $1-20$ and $66-85$.

For the terminal loop selection, the initial RNA pool was transcribed from $\sim 3 \times 10^{11}$ unique sequences. For the internal loop selections (R1, R2, and R3) the initial RNA pools were transcribed from $\sim 10^{13}$ unique sequences. Therefore, in all cases, multiple copies of each possible sequence were present at the beginning of the selection.

\section{DNA for high-throughput sequencing}

DNA for pyrosequencing (454 Life Sciences) was obtained by PCR amplification from 27 different selection pools including three Tryptophan selections, $70 \mathrm{~N}, 40$, and $60 \mathrm{~N}$ and $20 \mathrm{~N}$ (22). Fusion primers contained 454 Primer A fused to the T7 promoter sequence, and 454 Primer B fused to a $3^{\prime}$ primer specific for each selection. For selections that had common primers, 4-nt tags were attached to the $3^{\prime}$ end of the $3^{\prime}$ primer for identification of sequence pools. Each PCR preparation was PAGE purified, quantitated, and the final mixed DNA pool was further purified using Ultra Clean PCR cleanup kit (MO Bio Laboratories).

\section{Molecular modeling}

3D models were based on the secondary structures predicted by BayesFold (Knight et al. 2004a). NAB (Nucleic Acid Builder) (Macke and Case 1998) and Materials Studio (Accelrys, Inc.) were used to build the initial Trp sites. NAB was used to set up the main building blocks, modeling base pairs as regular A-form helices and unpaired regions (bulges or hairpin loops) as extended chains. Materials Studio (Accelrys, Inc.) was applied to manipulate and assemble the building units into the final 3D model, e.g., changing dihedral angles and introducing non-Watson-Crick pairs. For the RNA aptamer:tryptophan complex, several initial structures with tryptophan intercalated at different positions were built. The CHARMM27 force field (MacKerell et al. 1998; MacKerell and Banavali 2000) was used to describe interactions between RNA and tryptophan, and CHARMM (Brooks et al. 2009) was used to perform energy minimization, followed by molecular dynamics to reduce steric clashes. Because of the size of the simulated systems, the solvent effects were treated implicitly using the GBMV method (Lee et al. 2002, 2003), as implemented in 
CHARMM (Brooks et al. 2009) to reduce computation time. This method was recently refined and shown to provide results comparable with explicit solvent simulations for DNA (Chocholousova and Feig 2006). Values of the GBMV parameters were set according to previous studies on DNA (Chocholousova and Feig 2006a,b). Radii from the CHARMM force field were used.

Molecular dynamics simulations with the GBMV implicit solvent model were then started from the $3 \mathrm{D}$ model of the RNA aptamer (or RNA aptamer:tryptophan complex). In all simulations, the minimization of the initial structure was followed by an equilibration phase, in which the system was slowly heated up to room temperature. Based on previous studies (Chocholousova and Feig 2006a,b), the MD simulations were run with a 1 -fsec time step. Therefore, no holonomic constraints were applied. Langevin coupling with a friction coefficient of $50 \mathrm{ps}^{-1}$ was applied to all no-hydrogen atoms (Pastor et al. 1988).

MD simulations and Normal Mode Analysis were executed with CHARMM version c32b1 in combination with the MMTSB Tool Set (Feig et al. 2004). Results were inspected visually using VMD (Humphrey et al. 1996).

\section{Statistical analysis of conserved Trp motif elements}

In order to better understand the requirements for the loop B motif, we analyzed the deep-sequenced Trp 454 pool. Both mutagenic PCR, used in the $70 \mathrm{~N}$ selection, and sequencing errors artificially increase the number of selected Trp motifs. For meaningful statistics of the base composition of the Trp motif, we therefore needed to filter the sequence pool to its original sequences. We performed pairwise all-against-all sequence comparisons using clustalW2 (Larkin et al. 2007), and observed two distinct distributions for the maximal pairwise sequence identities of each sequence to any of the other sequences. In the case of the $70 \mathrm{~N}$ selection, one distribution was centered around $50 \%$, a second distribution was centered at $99 \%$, and almost no data points fell in
TABLE 1. Number of sequences analyzed in the 454 sequence pool

\begin{tabular}{lrrr}
\hline Statistic & $70 \mathrm{~N}$ & $40 / 60 \mathrm{~N}$ & $20 \mathrm{~N}$ \\
\hline Total number of sequences & 6432 & 17,398 & 13,303 \\
Unique origins & 5537 & 4774 & 2118 \\
Without PCR mutants & 707 & 2642 & 550 \\
Do fold CYA & 194 & 817 & - \\
Do not use primer & 95 & 368 & - \\
\hline
\end{tabular}

The last row gives the number of sequences analyzed for the refined Trp motif definition.

the $70 \%$ range (Fig. 8). The $50 \%$ peak obviously stems from different random origins. Conversely, the higher $99 \%$ peak reflects PCR mutations and sequencing errors. We concluded that a threshold of $80 \%$ sequence identity removes all but one sequence per unique origin. A similar consideration holds for the $40 \mathrm{~N}$ and $60 \mathrm{~N}$ selection, and to a lesser extent for the $20 \mathrm{~N}$ selection.

We performed further filtering by removing all sequences that cannot fold into a structure containing the CYA loop. For folding, we used a custom RNA structure prediction program that forces the motif to fold if it is compatible with standard base pairing. This procedure was based on the Locomotif program (Reeder and Giegerich 2007).

All isolates of the $20 \mathrm{~N}$ selection incorporate parts of the primer sequence into the motif. This reuse of the constant regions introduces bias. Consequently, the $20 \mathrm{~N}$ selection was not further considered. Some sequences in the other selections recruit primer bases to fold the motif, and were also excluded from the analysis. The total number of sequences in the initial pool and after each filtering step is given in Table 1. This final pool of sequences was used to define a revised Trp motif description, including the secondary loop.

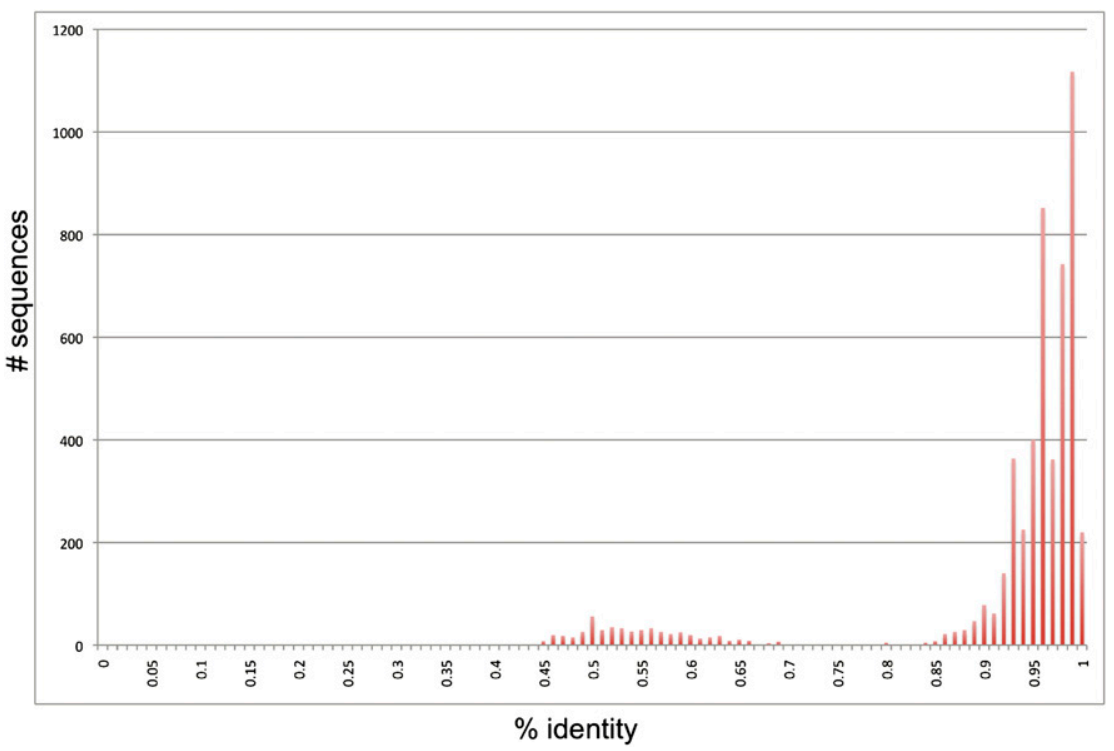

FIGURE 8. Plot of sequence similarities in the $70 \mathrm{~N} 454$ Trp pool. The $x$ axis shows pairwise sequence identity; the $y$ axis shows the number of sequences with that identity.

\section{ACKNOWLEDGMENTS}

We thank Jesse Stombaugh for comments on the manuscript. This research was funded by NASA Astrobiology Grant No. NNX08AP60G (to R.K.), NIH Bgrant No. R01 GM 48080 and its biocomplexity supplement (to M.Y.), and KONTAKT Grant No. ME09019 from the Ministry of Education, Youth and Sport of the Czech Republic (to J.C.); and through support from the Howard Hughes Medical Institute.

Received April 13, 2010; accepted June 22, 2010.

\section{REFERENCES}

Anderson PC, Mecozzi S. 2005a. Identification of a 14mer RNA that recognizes and binds flavin mononucleotide with high affinity. Nucleic Acids Res 33: 6992-6999.

Anderson PC, Mecozzi S. 2005b. Unusually short RNA sequences: Design of a 13-mer 
RNA that selectively binds and recognizes theophylline. $J$ Am Chem Soc 127: 5290-5291.

Bartel DP and Szostak JW. 1993. Isolation of new ribozymes from a large pool of random sequences. [see comment] Science 261: 14111418.

Bourdeau V, Ferbeyre G, Pageau M, Paquin B, Cedergren R. 1999. The distribution of RNA motifs in natural sequences. Nucleic Acids Res 27: 4457-4467.

Brooks BR, Brooks CL III, Mackerell AD Jr, Nilsson L, Petrella RJ, Roux B, Won Y, Archontis G, Bartels C, Boresch S., et al. 2009. CHARMM: The biomolecular simulation program. J Comput Chem 30: $1545-1614$.

Carothers JM, Oestreich SC, Davis JH, Szostak JW. 2004. Informational complexity and functional activity of RNA structures. J Am Chem Soc 126: 5130-5137.

Carothers JM, Oestreich SC, Szostak JW. 2006. Aptamers selected for higher-affinity binding are not more specific for the target ligand. J Am Chem Soc 128: 7929-7937.

Chocholousova J, Feig M. 2006a. Balancing an accurate representation of the molecular surface in generalized born formalisms with integrator stability in molecular dynamics simulations. J Comput Chem 27: 719-729.

Chocholousova J, Feig M. 2006b. Implicit solvent simulations of DNA and DNA-protein complexes: Agreement with explicit solvent vs experiment. J Phys Chem B 110: 17240-17251.

Ellington AD, Szostak JW. 1990. In vitro selection of RNA molecules that bind specific ligands. Nature 346: 818-822.

Feig M, Karanicolas J, Brooks CL 3rd. 2004. MMTSB Tool Set: Enhanced sampling and multiscale modeling methods for applications in structural biology. J Mol Graph Model 22: 377-395.

Harris ME, Nolan JM, Malhotra A, Brown JW, Harvey SC, Pace NR. 1994. Use of photoaffinity crosslinking and molecular modeling to analyze the global architecture of ribonuclease P RNA. EMBO J 13: 3953-3963.

Hofacker I, Fontana W, Stadler PF, Bonhoeffer LS, Tacker M, Schuster P. 1994. Fast folding and comparison of RNA secondary structures. Monatsh Chem 125: 167-188.

Humphrey W, Dalke A, Schulten K 1996. VMD: Visual molecular dynamics. J Mol Graph 14: 33-38.

Illangasekare M, Yarus M. 2002. Phenylalanine-binding RNAs and genetic code evolution. J Mol Evol 54: 298-311.

Kennedy R, Lladser ME, Yarus M, Knight R. 2008. Information, probability, and the abundance of the simplest RNA active sites. Front Biosci 13: 6060-6071.

Kennedy R, Lladser ME, Wu Z, Zhang C, Yarus M, De Sterck H, Knight R. 2010. Natural and artificial RNAs occupy the same restricted region of sequence space. RNA 16: 280-289.

Khvorova A, Lescoute A, Westhof E, Jayasena SD. 2003. Sequence elements outside the hammerhead ribozyme catalytic core enable intracellular activity. Nat Struct Biol 10: 708-712.

Kim N, Gan HH, Schlick T. 2007. A computational proposal for designing structured RNA pools for in vitro selection of RNAs. RNA 13: 478-492.

Knight R, Yarus M. 2003. Finding specific RNA motifs: Function in a zeptomole world? RNA 9: 218-230.

Knight R, Birmingham A, Yarus M. 2004a. BayesFold: Rational 2 degrees folds that combine thermodynamic, covariation, and chemical data for aligned RNA sequences. RNA 10: 1323-1336.
Knight R, Birmingham A, Yarus M. 2004b. BayesFold: Rational secondary folds that combine thermodynamic, covariation, and chemical data for aligned RNA sequences. RNA 10: 1323-1336.

Knight R, De Sterck H, Markel R, Smit S, Oshmyansky A, Yarus M. 2005. Abundance of correctly folded RNA motifs in sequence space, calculated on computational grids. Nucleic Acids Res 33: 5924-5935.

Larkin MA, Blackshields G, Brown NP, Chenna R, McGettigan PA, McWilliam H, Valentin F, Wallace IM, Wilm A, Lopez R, et al. 2007. Clustal W and Clustal X version 2.0. Bioinformatics 23: 2947-2948.

Laserson U, Gan HH, Schlick T. 2005. Predicting candidate genomic sequences that correspond to synthetic functional RNA motifs. Nucleic Acids Res 33: 6057-6069.

Lee MS, Salsbury FR Jr, Brooks CL III. 2002. Novel generalized Born methods. J Chem Phys 116: 10606-10614.

Lee MS, Feig M, Salsbury FR Jr, Brooks CL III. 2003. New analytic approximation to the standard molecular volume definition and its application to generalized Born calculations. J Comput Chem 24: 1348-1356.

Legiewicz M, Lozupone C, Knight R, Yarus M. 2005. Size, constant sequences, and optimal selection. RNA 11: 1701-1709.

Macke T, Case DA. 1998. Modeling unusual nucleic acid structures. In Molecular modeling of nucleic acids (ed. NB Leontis, J SantaLucia). American Chemical Society, Washington, DC.

MacKerell AD Jr, Banavali NK. 2000. All-atom empirical force field for nucleic acids: II. Application to molecular dynamics simulations of DNA and RNA in solution. J Comput Chem 21: 105-120.

MacKerell AD Jr, Bashford D, Bellott M, Dunbrack JD, Evanseck MJ, Field MJ, Fischer S, Gao J, Guo H, Ha S, et al. 1998. All atom empirical potential for molecular modeling and dynamics studies of proteins. J Phys Chem B 102: 3586-3616.

Majerfeld I, Yarus M. 2005. A diminutive and specific RNA binding site for L-tryptophan. Nucleic Acids Res 33: 5482-5493.

McCall MJ, Hendry P, Jennings PA. 1992. Minimal sequence requirements for ribozyme activity. Proc Natl Acad Sci 89: 5710-5714.

Pastor RW, Brooks BR, Szabo A. 1988. An analysis of the accuracy of langevin and molecular-dynamics algorithms. Mol Phys 65: 14091419.

Penedo JC, Wilson TJ, Jayasena SD, Khvorova A, Lilley DM. 2004. Folding of the natural hammerhead ribozyme is enhanced by interaction of auxiliary elements. RNA 10: $880-888$.

Quince C, Lanzen A, Curtis TP, Davenport RJ, Hall N, Head IM, Read LF, Sloan WT. 2009. Accurate determination of microbial diversity from 454 pyrosequencing data. Nat Methods 6: 639-641.

Reeder J, Giegerich R. 2007. Locomotif: From graphical motif description to RNA motif search. Bioinformatics 23: i392-i400.

Sabeti PC, Unrau PJ, Bartel DP. 1997. Accessing rare activities from random RNA sequences: The importance of the length of molecules in the starting pool. Chem Biol 4: 767-774.

Saksmerprome V, Roychowdhury-Saha M, Jayasena S, Khvorova A, Burke DH. 2004. Artificial tertiary motifs stabilize trans-cleaving hammerhead ribozymes under conditions of submillimolar divalent ions and high temperatures. RNA 10: 1916-1924.

Yarus M and Welch M. 2000. Peptidyl transferase: Ancient and exiguous. Chem Biol 7: R187-R190.

Yarus M, Widmann JJ, Knight R. 2009. RNA-amino acid binding: A stereochemical era for the Genetic Code. J Mol Evol 69: 406-429. 

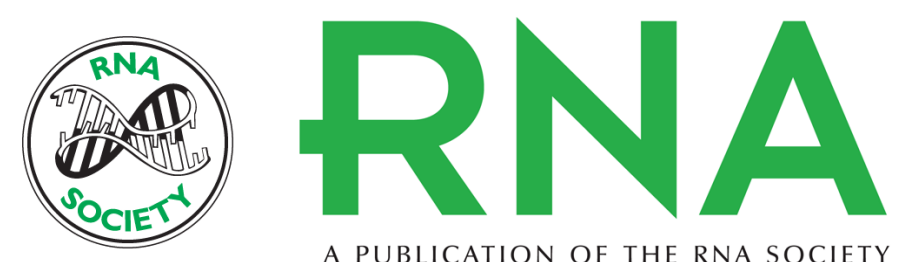

A PUBLICATION OF THE RNA SOCIETY

\section{Nucleotides that are essential but not conserved; a sufficient L-tryptophan site in RNA}

Irene Majerfeld, Jana Chocholousova, Vikas Malaiya, et al.

RNA 2010 16: 1915-1924 originally published online August 10, 2010

Access the most recent version at doi:10.1261/rna.2220210

\begin{aligned} & \hline References $\begin{array}{l}\text { This article cites } 40 \text { articles, } 10 \text { of which can be accessed free at: } \\ \text { http://rnajournal.cshlp.org/content/16/10/1915.full.html\#ref-list-1 }\end{array} \\ &$ Open Access Freely available online through the RNA Open Access option. \\ & License Freely available online through the RNA Open Access option. \\ & Email Alerting $\begin{array}{l}\text { Receive free email alerts when new articles cite this article - sign up in the box at the } \\ \text { top right corner of the article or click here. }\end{array} \\ &$\hline\end{aligned}

To subscribe to RNA go to:

http://rnajournal.cshlp.org/subscriptions 